\title{
A Study on the Perimenopausal Symptoms and Its Relevant Factors
}

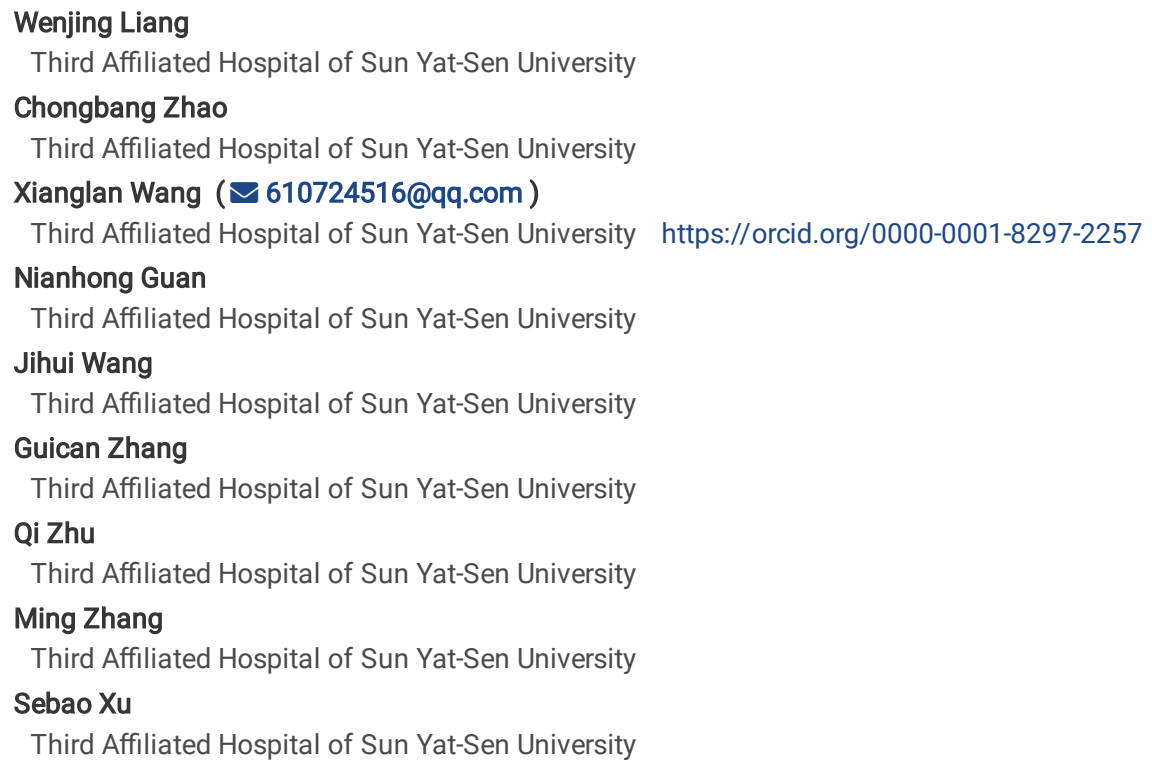




\section{Abstract}

Objective: To explore the relevant factors of perimenopausal symptoms.

Method: Community female subjects conforming to perimenopausal criteria were recruited through advertising. Modified Kupperman Index(KI) was used for evaluation of perimenopausal symptoms, Zung Self-Rating Depression Scale (SDS)for depressive symptoms and Zung Self-Rating Anxiety Scale (SAS)for anxiety symptoms. Eysenck Personality Questionnaire(EPQ) is used to evaluate the individual mental characteristics of subjects,including Extraversion(E), Neuroticism (N),Psychoticism(P), and Lie(L). Metacognition Questionnaire-30 (MCQ-30) is used to evaluate the metacognitive levels of subjects in the 5 important factors, including cognitive confidence(F1), positive beliefs about worry(F2), cognitive self-consciousness(f3), negative beliefs about uncontrollability of thoughts and danger(F4), and beliefs about the need to control thoughts (F5). Meanwhile, the levels of plasma gonadal hormones such as diol, testosterone and progesterone, are monitored. Pairwise correlation and linear regression are used to analyze the relevance between perimenopausal symptoms and the aforementioned factors.

Results: Among 66 perimenopausal female subjects, 40 (60.4\%) showed perimenopausal symptoms, with the most frequent being fatigue, joint and muscle pain , headache, dizziness, hypersensitivity and agitation. $N$ score $\left(\beta^{\prime}=0.419 \otimes P<0.001\right)$, SDS anxiety factor $\left(\beta^{\prime}=0.363 \otimes P<0.001\right)$ and $B M I \otimes \beta^{\prime}=0.244 \otimes P=0.001 \rrbracket$ are

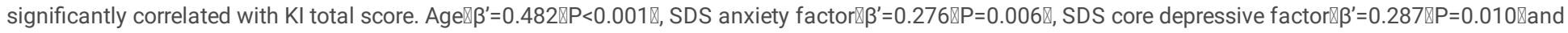
SDS anxiety factor $\varangle \beta^{\prime}=0.287 \otimes P=0.010 \rrbracket$ are significantly correlated with psychological symptoms. SAS standard score $\left(\beta^{\prime}=0.277 \rrbracket P=0.029\right)$ and $S D S\left(\beta^{\prime}=0.254 \rrbracket\right.$ $\mathrm{P}=0.045)$ are significantly correlated with somatic symptoms.

Conclusion: Emotional symptoms such as depression and anxiety are apparent. Perimenopausal females with Neuroticism personality, of elder age and higher BMI suffer from perimenopausal symptoms more severely.

\section{Introduction}

Perimenopause refers to the time period before and after the menopause of women. It starts from the inception of endocrine, biological and clinical menopausal features to one year after the last menstrual period[1].Perimenopause is a normal physiological transition period, during which the ovarian function of women gradually decline, the levels of sex hormones significantly decrease, the cycle of menstruation and volume of menses change and associated vasomotor symptoms (VMS) such as tidal fever and night sweating, somatic symptoms such as joints and muscle pain, dizziness, palpitation, paresthesia, and psychological symptoms such as insomnia, sensitive suspicious, emotional instability, collectively referred to as perimenopausal symptoms[1-2].

Perimenopausal symptoms not only affect the physical health of perimenopausal women, but also might be related to mental illnesses such as depression and anxiety. They constitute an important factor impacting the life quality of perimenopausal women and might further cause negative influences on their family members and the society as a whole. At present, the causes for perimenopausal symptoms have not been verified. Therefore, this study aims to conduct a comprehensive analysis of the causes from multiple perspectives including biology, clinical emotional symptoms, and psycho-social factors so as to further verify the major influential factors and provide scientific basis for clinical interventions in perimenopausal symptoms.

\section{Subjects And Methods}

\section{Research Subject}

This research proposal was approved by the Medical Ethics Committee of the Third Affiliated Hospital to Sun Yan-Sem University. The research subjects consist of 66 perimenopausal females recruited through ads by the researchers in the psychiatry department of this hospital. All the subjects signed a informed consent before participating in this study.

Inclusion criteria for subjects: 1 . Female, aged between 45 to 55. 2. received education of primary school or above, able to understand and complete the questionnaire and informed consent form independently. 3. conform to the basic standards of perimenopause formulated by the Stages of Reproductive Aging Workshop(STRAW): variability of the menstrual cycles (extended or shortened) $\nabla 7$ days (with/without change of menstrual blood volume)than normal or amenorrhea $₫ 12$ months. Exclusion criteria: 1. candidates had depression, hysteria or other mental illnesses before or had a family history of such conditions. 2. candidates were inflicted with severe physical illness, such as thyroid dysfunction, diabetes, adrenal dysfunction, and heart, liver or renal dysfunction. 3. candidates recently suffered from major mental stress, such as bereavement of kinsfolk, major loss of property, and serious marital or family issues.

\section{Research method}

2.1 Self-desgined questionnaires are used for the collection of demographic data, including age (year), height(m),weight(kg), educational level(years), menstrual conditions and marital status ( married or single, the latter including never married, divorced or widowed), number of kids, household income (RMB), work type (labour work, intellectual work or housewife), pressure of work (3 degrees including no or slight pressure, moderate pressure and heavy pressure).

\subsection{Evaluation Tools}

(1) Modified Kupperman Index, $\mathrm{KI}$ is used to evaluate the perimenopausal symptoms. The index contains 13 entries, with each graded 0,1,2,3 according to the severity of the subjects, with 0 denoting the lightest and 3 the most severe. There are three different categories of factors: factors of urinary system include urinary tract infection and discomfort with sexual activities (such as vaginal dryness, dyspareunia ); somatic factors include vasomotional symptoms such as 
hectic fever and night sweats, as well as paresthesia, palpitations, dizziness, headaches, ant crawling sensation, and joint and muscle pain; psychological factors include insomnia, fatigue, irritability and depression. Different questions have different weights, so the total score are the sum of the original score of each entry multiplied by their respective weight, with scores ranging from 0 to 63 . The severity of perimenopausal symptoms are evaluated according to the total scores they get: None: 0-6; Slight: 7-15; Medium:16-30; and Severe: 30 and above. The scores for each category of factors are the sum of all the scores of the entries included in this category ${ }^{[2]}$.

- Zung Self-rating Depression Scale (SDS) is used to evaluate the depression symptoms of the subjects. This assessment includes 20 items of statements, under each of which 4 choices:"never or seldom", "a little of the time", "good part of the time"and "most or all of the time"are available for the subjects to choose from that best describes their own conditions in the past week. The questions are scored on a scale of 1-4, with some questions reversely scored. The standard score of the scale equals to the total raw score multiplied by 1.25 . Romera et al (2008) divided the SDS by means of a factor analysis method into four factors: a core depressive factor (I); a cognitive factor (II); an anxiety factor (III) and a somatic factor (IV). Score of each factor=total score of all items/number of items ${ }^{[3,4]}$.

(3)Zung Self-rating Anxiety Scale (SAS) is used to evaluate the anxiety symptoms of the subjects. This assessment includes 20 items of statements, under each of which 4 choices:"never or seldom", "a little of the time", "good part of the time"and "most or all of the time"are available for the subjects to choose from that best describes their own conditions in the past week. The questions are scored on a scale of 1-4, with some questions reversely scored. The standard score of the scale equals to the total raw score multiplied by $1.25^{[5]}$.

(4)Eysenck Personality Questionnaire(EPQ)is adopted to evaluate the personal traits of the subjects. This study adopted the Chinese-revised version of the EPQ adult questionnaire ${ }^{[6]}$ to analyze the 4 factors of the subjects, including extraversion/introversion(E),neuroticism/stability(N),psychoticism/socialisation(P)and lie/social desirability $(\mathrm{L})$. The questionnaire contains 88 questions and the subjects are asked to answer "Yes" or "No" according to their own conditions.

(5) Metacognition Questionnaire-30 (MCQ-30): The short form revised by Wells ${ }^{[7]}$ in 2004 contains 30 items and is used to evaluate the metacognition of the

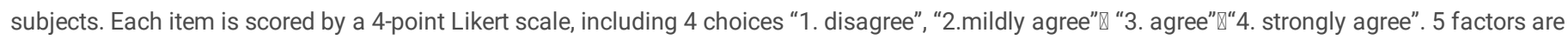
included:1.cognitive confidence(F1); 2.positive beliefs(F2); 3.cognitive self-consciousness (F3) 4.uncontrollability and danger(F4); 5. need to control thoughts(F5). The scores of each factor is obtained by adding up all the scores of the items involved in the factor.

\subsection{Detection of hormones}

1. time of detection :The time for blood sampling and questionnaire assessment for all the subjects up to the inclusion standards will be determined according to their menstrual conditions.

(1) For subjects with discernible menstrual cycles (subjects experience menstrual period every month or the cycle of menstruation is shorter than 2 months ) $\$ within 6 days after the menstruation. (2)For subjects with irregular menstrual cycles(subjects have no fixed menstrual cycles or experience over 2 months but less than 12 months of amenorrhea ):The detection time will be determined at the subjects' convenience.

(2)Detection method: $6 \mathrm{ml}$ of venous blood is sampled and centrifuged at $3000 \mathrm{r} / \mathrm{min}$ to separate plasma which is then stored for detection at minus 4 degrees celcius. Siemens ADVIA Centaur XP Immunoassay System is used to detect the estradiol, testosterone and progesterone in the plasma. All the detections are performed by professionals in the laboratory department of the Third Affiliated Hospital of Sun Yet-Sam University.

\section{Statistical Methods}

All statistical analyses are conducted by Statistical Product and Service Solutions(SPSS『Windows version 16.0. All the data are input into SPSS to form databases. The correlation analysis of symptoms of $\mathrm{KI}$ and all factors is done by Spearman correlation and the correlation analysis of $\mathrm{KI}$ factors and all factors is done by Pearson correlation. The dependant variables are the KI total score and the scores of each factor. Statistically significant variables in the above-mentioned two correlation analyses will be used as independent variables and analyzed through multiple linear regression(MLR). Step by step method is adopted to screen the variables, with both inclusion and exclusion P values being 0.05. The standard Alpha level =0.05.

Results

\section{Demographic Characteristics}

This study included 66 perimenopausal females up to the inclusion standards, aged from 45 to 52 , with an average age of $47.5 \pm 2.01$. Educational levels of subjects range from 5 to 12 years,averaging $7.78 \pm 1.83$ years. Marital status: 54 married (81.8\%), 8 divorced (12.2\%),3 widowed(4.5\%) and 1 single(1.5\%). Number of children: 44 subjects(66.7\%) have 2 children or above, 20 subjects(30.3\%) have 1 child, 2 subjects(3.0\%) have no children. Monthly income: 2 subjects(3.0\%) below 1000 RMB, 31 subjects(47.0\%) 1000 to 3000RMB, 23 subjects(34.8\%) 3000 to 5000 RMB, 10 subjects(15.2\%) above $5000 \mathrm{RMB}$. Work type: manual labour, 32 subjects(48.5\%); mental labour, 4 subjects(6.1\%); housewives, 30 subjects(45.5\%);Work pressure: none or slight, 16 subjects (24.2\%); medium, 45 subjects(68.2\%), heavy, 5 subjects (7.6\%). BMl of subjects range from $9.53 \mathrm{~kg} / \mathrm{m} 2 \sim 32.05 \mathrm{~kg} / \mathrm{m} 2$, averaging $\varangle 24.24 \pm 2.84 \llbracket \mathrm{kg} / \mathrm{m} 2$. Menstrual status: 61 subjects (92.4\%) experienced a change of menstrual periods longer than 7 days but shorter than 2 months. 5 subjects had menstrual extension for over 2 months and amenorrhea for less than 12 months. 


\section{Perimenopausal symptoms}

Among the investigated subjects, 40 (60.4\%) showed menopausal symptoms, with the most frequent being fatigue, joint and muscle pain, headache, dizziness, hypersensitivity and agitation. Among the 40 females showing perimenopausal symptoms, 31 had slight symptoms, 9 had medium or heavy symptoms.

3. correlation analysis of sex hormones and perimenopausal symptoms

The correlation between the level of sex hormones and perimenopausal symptoms are indicated in Table 1. The level of estradiol is in negative correlation only with arthralgia $(r=-0.235, P=0.058)$; testosterone is in low positive correlation with depressive and suspicious symptoms $(r=0.334 \rrbracket P=0.006)$.

Progesterone is in low positive association with vertigo symptoms $₫ r=0.325 \rrbracket P=0.008 \rrbracket$, and in weak negative correlation with fatigue symptoms $\varangle r=-0.295 \rrbracket P$ $=0.016 \rrbracket$, and in weak negative correlation with sexual discomfort symptoms $₫ r=-0.235 \bigotimes P=0.057 \rrbracket$.

\section{Correlation analysis between perimenopausal symptoms and depressive and anxious symptoms}

The correlation between depressive and anxious symptoms and perimenopausal symptoms are indicated in Table 1. In general, the standard score, the core factor and the somatic factor of depression are in low or weak positive correlation to perimenopausal symptoms $(0.235 \leq r \leq 0.386)$; the core factor and anxiety factor are in moderate positive correlation to total score of $\mathrm{KI}(r=0.510 \otimes P=0.008)$ and they are in low or weak positive correlation to other perimenopausal symptoms; there is no significant correlation between the cognition factor and the perimenopausal symptoms. The anxiety score is in low or weak positive correlation to the perimenopausal symptoms $₫ 0.289 \leq r \leq 0.447 \rrbracket$.

5. Correlation analysis of perimenopausal symptoms with general information, meta-cognition, personal traits \see Table 2】

The personal and family traits of female subjects have certain impacts on their perimenopausal symptoms. Age is in moderate positive correlation with urogenital symptoms $\ r=0.517 \rrbracket P=0.008 \rrbracket$ and sexual discomfort $(r=0.522 \bigotimes P=0.008)$. Number of children, BMI, work pressure and household income are in low or weak positive correlation $₫ 0.255 \leq r \leq 0.362 \rrbracket$ with perimenopausal symptoms. Years of education is in weak negative correlation $\varangle r=-0.248 \rrbracket P=0.008 \rrbracket$ with headache.

Among the personality factors, neuroticism/stability $(\mathrm{N})$ is in moderate positive correlation $(r=0.550 \rrbracket P=0.008 \rrbracket$ with $\mathrm{KI}$ total score and is in low or weak positive correlation $\varangle 0.280 \leq r \leq 0.491$ with other perimenopausal symptoms. Psychoticism/socialisation $(P)$ is in weak positive correlation with sexual discomfort $₫ r=$ $0.275 \rrbracket P=0.008 \rrbracket$ and in low positive correlation with fatigue symptoms $₫ r=0.311 \rrbracket P=0.008 \rrbracket$. Lie/social desirability $(\mathrm{L})$ is in low or weak negative correlation with perimenopausal symptoms $\mathbb{0}-0.478 \leq r \leq-0.254 \rrbracket$. Neuroticism/stability $(\mathrm{N})$ is not significantly correlated with perimenopausal symptoms statistically.

In metacognition, cognitive self-consciousness, positive beliefs and uncontrollability and danger are in low or weak positive correlation $\varangle 0.250 \leq r \leq 0.329 \rrbracket$ with perimenopausal symptoms and need to control thoughts are only in weak negative correlation with sexual discomfort. Cognitive self-consciousness is not significantly correlated with perimenopausal symptoms statistically.

\section{Multivariate regression analysis of factors affecting perimenopausal symptoms}

The results of multivariate regression analysis are shown in Table 3. neuroticism/stability $(\mathrm{N}) \otimes \beta^{\prime}=0.419 \bigotimes P<0.001 \rrbracket$, anxiety factor $₫ \beta^{\prime}=0.363 \rrbracket P<0.001 \rrbracket$ and $\mathrm{BMI}$ $\square \beta^{\prime}=0.244 \rrbracket P=0.001 \rrbracket$ are in significant correlation with $\mathrm{KI}$ total score.Age $\otimes \beta^{\prime}=0.482 \otimes P<0.001 \rrbracket$, anxiety factor $\otimes \beta^{\prime}=0.276 \rrbracket P=0.006 \rrbracket$, core factor $\varangle \beta^{\prime}=0.208 \rrbracket P=0.026 \rrbracket$ and neuroticism/stability $(\mathrm{N}) \otimes \beta=0.226 \otimes P=0.025 \bigotimes$ are in significant correlation with the genitourinary symptoms. Neuroticism/stability $(\mathrm{N}) \otimes \beta^{\prime}=0.247 \otimes P=0.048 \rrbracket$, Lie/social desirability $(\mathrm{L}) \otimes \beta^{\prime}=-0.272 \rrbracket P=0.024 \rrbracket$, and anxiety factor $\otimes \beta^{\prime}=0.287 \rrbracket P=0.010 \rrbracket$ are significantly correlated with psychological symptoms. Anxiety standard score $\otimes \beta^{\prime}=0.277 \rrbracket P=0.029 \rrbracket$ and anxiety factor $\otimes \beta^{\prime}=0.254 \rrbracket P=0.045 \rrbracket$ are significantly correlated with somatic symptoms.

\section{Discussion}

Perimenopausal symptoms have a significant impact on the physical and mental health of women. This study shows that the incidence rate of perimenopausal symptoms reaches $60.9 \%$, with that of moderate to serious symptoms at $13.6 \%$. Fatigue, joint and muscle pain, headache, dizziness, hypersensitivity and agitation are the most frequent perimenopausal symptoms. The occurrence of perimenopausal symptoms are significantly correlated with depression, anxiety, personality and psychosocial factors, with varying correlational factors present in different symptoms.

The changes of sex hormone levels during perimenopause are the significant causes for perimenopausal symptoms. Estrogen receptors (ER) can be found in the cytomembranes of over 400 parts of tissue or organs in females, in which degenerative or metabolic changes can be resulted from reduced secretion of estrogen. Such changes may cause in females during perimenopause or after menopause imbalances in multiple systems, including the nervous system, psychological system and neuroendocrine system, which further leads to a series of symptoms of mental and endocrine disorders. [8]

In addition, symptoms of the genitourinary and neuropsychiatric systems are not only related to hormone levels in vivo, but also closely related to physiological aging [9]. Therefore, age is also an important cause of perimenopausal symptoms. In this study, there was no significant correlation between sex hormones and perimenopausal symptoms, which may be related to false negative results caused by small sample size. Despite that, the researcher still observed a negative correlation between estradiol levels and perimenopausal symptoms, suggesting that the lower the estrogen level, the more apparent the perimenopausal symptoms.

Depression is a common psychological symptom, with high incidence rate both in perimenopausal females and among normal people. A research by Zhang,et al [10] found that $22.5 \%$ of 542 healthy workers displayed depressive symptoms. Comstock, et al [11] surveyed 1173 residence, among which $20 \%$ displayed 
depressive symptoms. Female subjects with past infliction of perimenopause have markedly higher incidence of depression than that of normal people. A survey by Beijing Maternity and Child Care Center[12] indicated an incidence rate of depression as high as $59.45 \%$ for perimenopausal females, with $17.17 \%$ experiencing severe depressive symptoms. Gao, et al [13] reported on the mental status of 2000 females of 40 to 60 years old, indicating a $30.3 \%$ of women were showing depressive symptoms. Anxiety and depression often happen simultaneously. The majority of perimenopausal women would experience anxiety, which is believed to be related to family background, life pressure, and social factors, etc.

An epidemiological survey in Beijing area[14] found that major factors related to depression and anxiety of perimenopausal women include: financial difficulties, lack of social support, dissatisfaction with family, pressure from children's schooling, sexual discomfort during perimenopause, tidal fever and sweating,etc. Another research found that the decrease of estrogen level during perimenopause is also an important factor that causes depression and anxiety disorders[15]. Applying estradiol therapy to perimenopausal women in the short term can alleviate depressive symptoms[16].

This research also found that perimenopausal symptoms are closely related with depression and anxiety disorders. More apparent genitourinary, mental and somatic symptoms during perimenopause mean higher incidence rate of depression and anxiety. These results indicate that perimenopausal symptoms are the major factors impacting on depression and anxiety barriers.

The relations between personality traits and perimenopausal symptoms receive relatively less attention from researchers. This study shows that among perimenopausal women, those with more apparent emotional instability are easier to have genitourinary, mental and somatic symptoms, while those with better social desirability would suffer from less mental symptoms. All the research results indicate that personality traits are significant factors holding perimenopausal symptoms.

Metacognition is the cognition of cognition. It is embodied in the planning, supervision and regulation of the cognitive processes. The function of metacognition in the occurrence and development of mental illnesses are gaining more attention day by day. The self-regulatory executive function model believes that mental patients with depressive disorder have non-adaptive meta-cognitive features, while intervention with the metacognition can effectively alleviate depressive disorder. However, there are currently limited research on perimenopausal symptoms and metacognition. The findings of this research indicate that mental patients with depressive disorder demonstrate non-adaptive meta-cognitive features, while intervention into the metacognition can effectively alleviate depressive disorder[19].

This research revealed that subjects with more apparent perimenopausal symptoms would have less cognitive self-consciousness(F1) and more uncontrollability and danger(F4), and are more likely to have positive beliefs $\varangle \mathrm{F} 2 \bigotimes$ about depression during cognition and have stronger need to control thoughts(F5).

In terms of demographic data, age, BMI, number of children, and work pressure are shown to be in positive correlation with perimenopausal symptoms. Those factors might increase the psychological pressure of perimenopausal females, which is still to be verified through further research. On the other hand, subjects with higher educational background suffer less from perimenopausal symptoms. It is indicated that in our group of subjects, higher educational background may allow subjects to adopt a more proper attitude towards perimenopausal symptoms.

In conclusion, The occurrence of perimenopausal symptoms are closely related with factors such as depressive symptoms, anxiety symptoms, personality, age,and BMI, etc.

\section{Disclosure Statement}

The authors declare that they have no conflicts of interest. The study was supported by the Science and Technology Program of Guangdong, China (2010B031600309 to Xianglan Wang).

\section{Reference}

[1] Brambilla DJ, McKinlay SM, Johannes CB. Defining the perimenopause for application in epidemiologic investigations. Am J Epidemiol. 1994 15;140(12):1091-5.

[2] Shaver JL1, Paulsen VM. Sleep, psychological distress, and somatic symptoms in perimenopausal women. Fam Pract Res J. 1993;13(4):373-84.

[3] Harlow S D, Gass M, Hall J E, et al. Executive summary of the Stages of Reproductive Aging Workshop +10: addressing the unfinished agenda of staging reproductive aging[J]. Climacteric, 2012,15(2):105-114.

[4] Tao M, Shao H, Li C, et al. Correlation between the modified Kupperman Index and the Menopause Rating Scale in Chinese women[J]. Patient Prefer Adherence, 2013,7(3):223-229.

[5] Romera I, Delgado-Cohen H, Perez T, et al. Factor analysis of the Zung self-rating depression scale in a large sample of patients with major depressive disorder in primary care[J]. BMC Psychiatry, 2008,8(1):4.

[6] Yaoxiao G. Revisions to the Eysenck personality questionnaire(EPQ) [J]. Psychological Science, 1984(4):13-20.

[7] Wells A, Cartwright-Hatton S. A short form of the metacognitions questionnaire: properties of the MCQ-30[J]. Behav Res Ther, 2004,42(4):385-396.

[8] Burger H, Woods NF, Dennerstein L et al. Nomenclature and endocrinology of menopause and perimenopause [J]. Expert Rev Neurother. 2007,7(11):35-43 
[9] Jiayun Q,Xiaoyan L, Liping W, Yan W.The correlation between levels of serum sex hormones and blood fat with perimenopausal symptoms .Maternal and Child Health Care of China,2013,28(9):108-111.

[10] Mingyuan Zhang, Fumin Ren.The investigation of depressive symptoms and the Application of CES2D in normal population. Chinese Journal of Psychiatry, 1987,20:671.

[11] Comstock GM, Helsing KJ. Symptom of two communities. Psycho Med, 1976,6:551.

[12] Xiangyan Ruan,Yamei Cui,Juan Du,et al.Prevalence of climacteric symptoms comparing perimenopausal and postmenopausal Chinese women.J Psychosom Obstet Gynecol.2017;38:161-169.

[13] Xiaoling Gao,Zhewei Wang,Jiazeng Hua, et al. A research on the occurence and influencing factors of depressive symptoms in menopausal women [J] Maternal and child health care of China,1998,13(5):294-296.

[14] Ying Li,Qi Yu,Liangkun Ma,Zhengyi Sun. The correlation between social support and depressive and anxiety symptoms of perimenopausal women. [J].Chinese journal of clinical obstetrics and gynecology, 2009,10(2):116-118.

[15] Lokuge S, Frey BN, Foster JA, et al. Depression in women: windows of vulnerability and new insights into the link between estrogen and serotonin. J Clin Psychiatry. 2011;72(11):e1563-9.

[16] Soares CN,Almeida OP,Joffe H,et al. Efficacy of estradiol for the treatment of depressive disorders in perimenopausal women:adoubleblind,randomized,placebo-controlled trial.Archi Gen Psychiatry,2001,58(6):529-534.

[17] Janet Metcalfe区Arthur P Shimamura. Metacognition: Knowing About Knowing. MIT Press, Cambridge, MA,1994.

[18] Matthews G, Wells A. Rumination, depression, and metacognition: The S-REF model [M]//Papageorgiou C, Wells A. Depressive Rumination: Nature, Theory and Treatment. Hoboken: JohnWiley \& Sons, 2004: 125-151.

[19] Normann N, van Emmerik AA, Morina N. The efficacy of meta囚cognitive therapy for anxiety and depression: a meta-analytic review [J]. Depress Anxiety, 2014, 31(5): 402-411.

\section{Tables}

Table 1. Scores of KI and the correlational analysis between scores of KI and scores of sex hormones, SDS and SAS. 


\begin{tabular}{|c|c|c|c|c|c|c|c|c|c|c|}
\hline & \multirow[t]{2}{*}{ score } & \multicolumn{3}{|c|}{ Sexual hormones $\square r \llbracket$} & \multicolumn{5}{|c|}{ SDS score $\llbracket r \rrbracket$} & \multirow{2}{*}{ 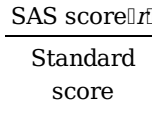 } \\
\hline & & estradiol & testosterone & progesterone & $\begin{array}{l}\text { Standard } \\
\text { score }\end{array}$ & $\begin{array}{l}\text { Core } \\
\text { factor }\end{array}$ & $\begin{array}{l}\text { Cognition } \\
\text { factor }\end{array}$ & $\begin{array}{l}\text { Anxiety } \\
\text { factor }\end{array}$ & $\begin{array}{l}\text { Somatic } \\
\text { factor }\end{array}$ & \\
\hline Total score & $8.91 \pm 6.04$ & -0.122 & 0.038 & -0.130 & $0.386^{\mathrm{b}}$ & 0.235 & 0.149 & $0.510^{\mathrm{b}}$ & $0.255^{\mathrm{a}}$ & $0.447^{\mathrm{b}}$ \\
\hline Genito-urinary symptoms & $1.33 \pm 1.62$ & -0.154 & -0.025 & -0.178 & $0.367^{b}$ & $0.264^{\mathrm{a}}$ & 0.181 & $0.376^{\mathrm{b}}$ & $0.298^{\mathrm{a}}$ & $0.306^{\mathrm{a}}$ \\
\hline Mental symptoms & $2.80 \pm 2.64$ & -0.009 & -0.117 & -0.241 & $0.268^{\mathrm{a}}$ & 0.148 & 0.031 & $0.439^{\mathrm{b}}$ & 0.112 & $0.321^{\mathrm{b}}$ \\
\hline somatic symptoms & $4.77 \pm 3.53$ & -0.127 & 0.176 & 0.020 & $0.291^{\mathrm{a}}$ & 0.171 & 0.148 & $0.371^{\mathrm{b}}$ & 0.216 & $0.384^{\mathrm{b}}$ \\
\hline \multicolumn{11}{|c|}{ Percentages of entries with score $\geq 1$} \\
\hline Urinary tract infection & 9.1 & -0.003 & 0.197 & 0.033 & 0.091 & 0.019 & -0.093 & $0.326^{\mathrm{d}}$ & 0.150 & 0.146 \\
\hline Sexual discomfort & 47.0 & -0.181 & -0.108 & -0.235 & $0.392^{\mathrm{d}}$ & $0.301^{\mathrm{c}}$ & 0.204 & $0.366^{\mathrm{d}}$ & $0.311^{\mathrm{c}}$ & $0.430^{\mathrm{d}}$ \\
\hline $\begin{array}{l}\text { tidal fever and night } \\
\text { sweatings }\end{array}$ & 36.4 & -0.124 & 0.081 & 0.016 & 0.176 & 0.141 & 0.038 & $0.294^{\mathrm{c}}$ & 0.212 & 0.084 \\
\hline Palpitation & 28.8 & -0.105 & 0.017 & 0.030 & $0.257^{\mathrm{c}}$ & 0.210 & 0.148 & $0.288^{\mathrm{c}}$ & 0.024 & $0.289^{c}$ \\
\hline Dizziness & 54.5 & 0.202 & 0.210 & $0.325^{\mathrm{d}}$ & 0.041 & 0.045 & 0.056 & -0.028 & 0.046 & 0.152 \\
\hline Headache & 57.5 & -0.033 & 0.049 & -0.040 & 0.227 & 0.166 & 0.084 & 0.132 & 0.114 & $0.320^{\mathrm{d}}$ \\
\hline Paresthesia & 42.4 & -0.087 & 0.117 & -0.013 & 0.223 & 0.079 & 0.180 & 0.238 & 0.140 & $0.319^{d}$ \\
\hline Ant crawling sensation & 22.7 & -0.072 & 0.128 & -0.085 & 0.033 & -0.113 & -0.034 & $0.315^{\mathrm{c}}$ & 0.034 & $0.422^{\mathrm{d}}$ \\
\hline Joint and muscle pain & 60.6 & -0.235 & 0.169 & -0.189 & $0.383^{\mathrm{d}}$ & $0.262^{\mathrm{c}}$ & 0.192 & 0.202 & 0.183 & $0.378^{\mathrm{d}}$ \\
\hline insomnia & 25.8 & -0.013 & -0.077 & -0.093 & $0.279^{c}$ & $0.250^{\mathrm{c}}$ & 0.111 & $0.314^{\mathrm{c}}$ & 0.215 & 0.151 \\
\hline fatigue & 63.6 & 0.006 & -0.073 & $-0.295^{\mathrm{c}}$ & $0.327^{d}$ & $0.323^{\mathrm{d}}$ & 0.041 & 0.202 & $0.306^{\mathrm{c}}$ & $0.359^{d}$ \\
\hline $\begin{array}{l}\text { Depression and } \\
\text { suspicion }\end{array}$ & 15.2 & 0.002 & $0.334^{\mathrm{d}}$ & 0.043 & 0.049 & 0.030 & -0.090 & $0.260^{\mathrm{c}}$ & -0.013 & 0.168 \\
\hline Irritability & 50.0 & -0.046 & -0.161 & -0.092 & 0.197 & 0.080 & 0.006 & $0.352^{\mathrm{d}}$ & -0.096 & $0.291^{\mathrm{c}}$ \\
\hline \multicolumn{11}{|l|}{ Severity } \\
\hline No symptoms & $26(39.4 \%)$ & & & & & & & & & \\
\hline Slight symptoms & $31(47.0 \%)$ & & & & & & & & & \\
\hline Intermediate to Severe & $9(13.6 \%)$ & & & & & & & & & \\
\hline
\end{tabular}


according to Spearman correlation analysis.

Table 2 Correlation analysis (r) between scores of KI and other factors and scores of general information, EPQ and MCQ.

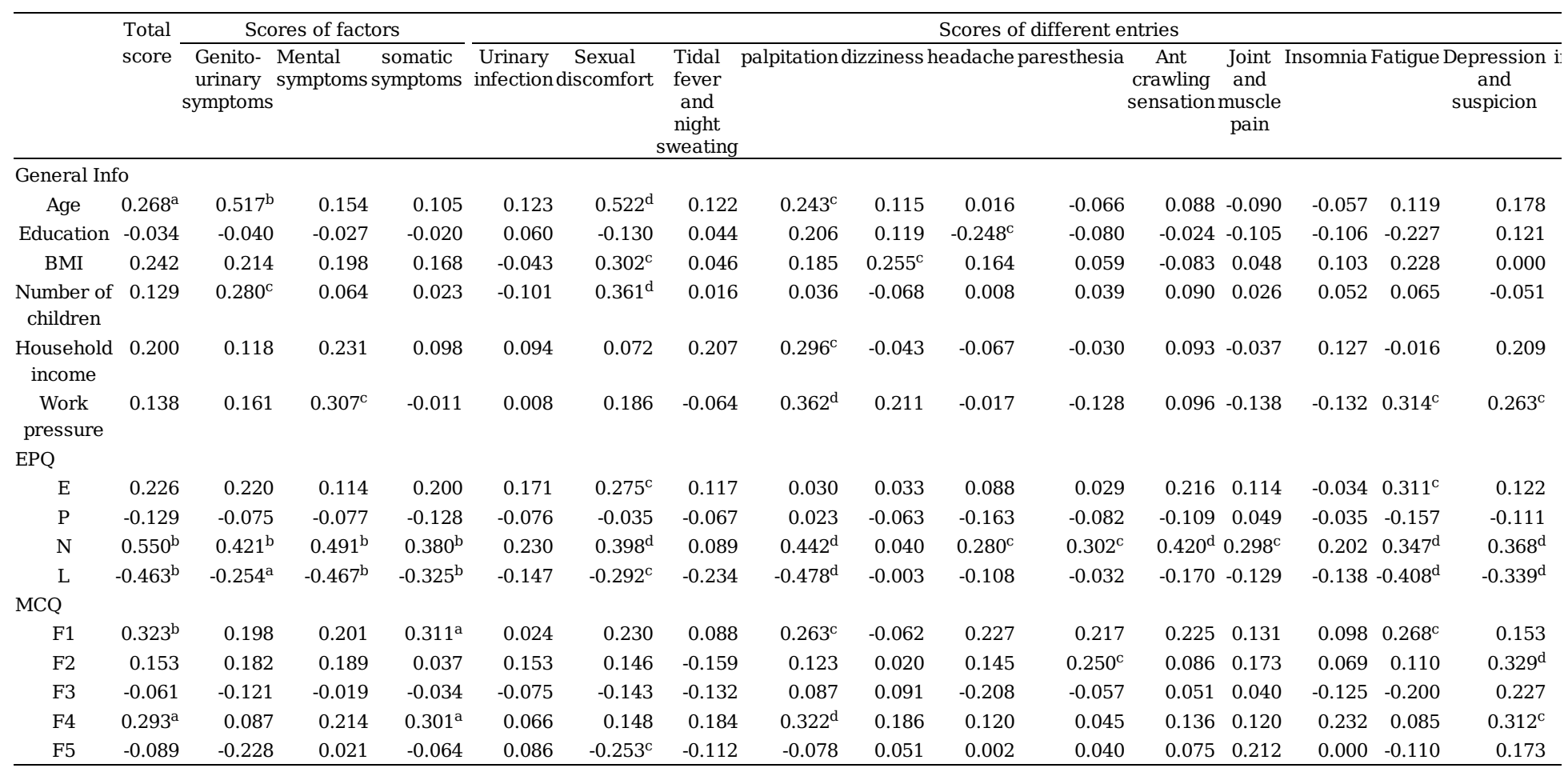




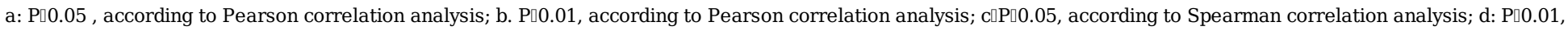
according to Spearman correlation analysis.

Table 3. Regression analysis between total score of KI and scores of other factors

\begin{tabular}{|c|c|c|c|c|c|c|c|}
\hline dependant variable & Independent variable & $\beta$ & $\beta^{\prime}$ & $P$ & Adjusted $R^{2}$ & $F$ & $P$ \\
\hline \multirow[t]{3}{*}{ Total score of KI } & EPQ N & 0.276 & 0.419 & $\square 0.001$ & 0.449 & 18.640 & $\square 0.001$ \\
\hline & SDS anxiety factors & 3.487 & 0.363 & $\square 0.001$ & & & \\
\hline & BMI & 0.519 & 0.244 & 0.010 & & & \\
\hline \multirow[t]{4}{*}{ Score of genitourinary symptoms } & Age & 0.374 & 0.482 & $\square 0.001$ & 0.471 & 15.478 & $\square 0.001$ \\
\hline & SDS anxiety factors & 0.712 & 0.276 & 0.006 & & & \\
\hline & EPQ N & 0.040 & 0.226 & 0.025 & & & \\
\hline & SDS core factors & 0.609 & 0.208 & 0.026 & & & \\
\hline \multirow[t]{3}{*}{ score of mental symptoms } & EPQ N & 0.071 & 0.247 & 0.048 & 0.344 & 12.348 & $\square 0.001$ \\
\hline & SDS anxiety factors & 1.207 & 0.287 & 0.010 & & & \\
\hline & EPQ L & -0.077 & -0.272 & 0.024 & & & \\
\hline \multirow[t]{2}{*}{ score of somatic symptoms } & SAS standard score & 0.111 & 0.277 & 0.029 & 0.175 & 7.907 & 0.001 \\
\hline & SDS anxiety factors & 1.425 & 0.254 & 0.045 & & & \\
\hline
\end{tabular}

